\title{
SOME CONVEXITY THEOREMS FOR MATRICES
}

\author{
by P. A. FILLMORE and J. P. WILLIAMS \\ (Received 8 October, 1969)
}

Introduction. The numerical range of a bounded linear operator $A$ on a complex Hilbert space $H$ is the set $W(A)=\{(A f, f):\|f\|=1\}$. Because it is convex and its closure contains the spectrum of $A$, the numerical range is often a useful tool in operator theory. However, even when $H$ is two-dimensional, the numerical range of an operator can be large relative to its spectrum, so that knowledge of $W(A)$ generally permits only crude information about $A$. P. R. Halmos [2] has suggested a refinement of the notion of numerical range by introducing the $k$-numerical ranges

$$
W_{k}(A)=\left\{\frac{1}{k} \operatorname{tr}(P A): P=\text { projection of rank } k\right\}
$$

for $k=1,2,3, \ldots$ It is clear that $W_{1}(A)=W(A)$. C. A. Berger [2] has shown that $W_{k}(A)$ is convex.

In Section 1 of this paper we obtain a few additional results about $k$-numerical ranges, including a description of $W_{k}(A)$ for normal matrices $A$. In Section 2 we introduce another generalized numerical range $\mathscr{W}(A)$, which by definition consists of the diagonals of all matrices that are unitarily equivalent to $A$. A theorem of Horn [3] shows that $\mathscr{W}(A)$ is convex if $A$ is a Hermitian matrix; this can fail for normal matrices of order $\geqq 3[5,7]$. By computing the convex hull of $\mathscr{W}(A)$ for normal matrices $A$, we obtain a generalization of a result of $\mathrm{F}$. John [4]. Finally, in Section 3 we exploit the connection between $\mathscr{W}(A)$ and $k$-numerical ranges to obtain a simple proof of Horn's result.

1. $k$-numerical ranges. Throughout the paper $H$ is a complex Hilbert space of dimension $n<\infty$, and $A$ is a linear operator on $H$. We begin by listing some elementary properties of $W_{k}(A)$.

THEOREM 1.1. For any operator $A$ on $H$,

(i) $W_{k}(A)$ is convex and compact.

(ii) $(n-k) W_{n-k}(A)=\operatorname{tr}(A)-k W_{k}(A) \quad(k=1,2, \ldots, n-1)$.

(iii) $W_{k}\left(U^{-1} A U\right)=W_{k}(A)$ if $U$ is unitary.

(iv) $W_{n}(A)=(1 / n) \operatorname{tr}(A), W_{1}(A)=W(A)$.

(v) $W_{k}(A)$ contains each normalized sum

$$
\frac{1}{k}\left(\lambda_{i_{1}}+\lambda_{i_{2}}+\ldots+\lambda_{i_{k}}\right)
$$

of eigenvalues of $A$.

(vi) $W_{k+1}(A) \subset W_{k}(A) \quad(k=1,2, \ldots, n-1)$.

Proof. As mentioned in the Introduction, the convexity of $W_{k}(A)$ was proved by Berger. The rest of (i) follows from the continuity of the trace and the compactness of the set of rank 
$k$ projections. Statements (ii), (iii), and (iv) are clear from the definition. Assertion (v) is an immediate consequence of (iii) and the fact that $A$ is unitarily equivalent to a matrix in triangular form. The inclusion in (vi) will follow from Theorem (1.2) and the fact that if $O \leqq X \leqq I$ and $\operatorname{tr}(X)=k+1$, then

$$
\frac{1}{k+1} \operatorname{tr}(X A)=\frac{1}{k} \operatorname{tr}(Y A)
$$

where

$$
Y=\frac{k}{k+1} X .
$$

Note that if $k=1$, then (v) reduces to the familiar fact that the numerical range contains the spectrum.

THEOREM 1.2 .

$$
W_{k}(A)=\left\{\frac{1}{k} \operatorname{tr}(X A): 0 \leqq X \leqq I, \operatorname{tr}(X)=k\right\} .
$$

In order to prove Theorem 1.2 we need two lemmas.

LeMMA 1.3. Let $P_{k}$ be the set of $n$-tuples $\left\langle p_{1}, p_{2}, \ldots, p_{n}\right\rangle$ satisfying $0 \leqq p_{i} \leqq 1, \sum_{i} p_{l}=k$. Then $P_{k}$ is compact and convex, and the set $\operatorname{Ext}\left(P_{k}\right)$ of extreme points of $P_{k}$ consists of all vectors with $k$ coordinates equal to 1 and the rest equal to 0 .

Proof. If $p=\left\langle p_{1}, p_{2}, \ldots, p_{n}\right\rangle$ belongs to $P_{k}$ and if $0<p_{1}<1,0<p_{i}<1$ for some $i \neq 1$, because $k$ is an integer. If $\varepsilon=\min \left\{p_{1}, p_{i}, 1-p_{1}, 1-p_{i}\right\}$, then $p=\frac{1}{2}\left(p^{\prime}+p^{\prime \prime}\right)$, where

$$
\begin{aligned}
p^{\prime} & =\left\langle p_{1}-\varepsilon, p_{2}, \ldots, p_{i}+\varepsilon, \ldots, p_{n}\right\rangle, \\
p^{\prime \prime} & =\left\langle p_{1}+\varepsilon, p_{2}, \ldots, p_{i}-\varepsilon, \ldots, p_{n}\right\rangle .
\end{aligned}
$$

Since $p^{\prime}, p^{\prime \prime}$ belong to $P_{k}$, it follows that $p$ is not an extreme point of $P_{k}$. The same argument clearly works for the other coordinates of $p$. Hence if $p$ is an extreme point, then each $p_{t}$ is either 0 or 1 . Thus exactly $k$ coordinates of $p$ equal 1 and the others are 0 . Conversely, it is clear that each such vector is an extreme point of $P_{k}$.

LEMMA 1.4. The convex hull of the set of Hermitian projections of rank $k$ consists of those operators $X$ satisfying $O \leqq X \leqq I, \operatorname{tr}(X)=k$.

Proof. Suppose $O \leqq X \leqq I$ and $\operatorname{tr}(X)=k$. By the spectral theorem we can write $X=\sum_{i=1}^{n} \lambda_{i} E_{i}$, where the $E_{i}$ are mutually orthogonal projections of rank 1 , and the $\lambda_{l}$ are the eigenvalues of $X$. Then $0 \leqq \lambda_{i} \leqq 1$ and $\sum_{i=1}^{n} \lambda_{i}=k$, and Lemma 1.3 implies that $X$ is a convex combination of projections of rank $k$. 
The converse follows from the obvious fact that the set of operators $X$ satisfying $O \leqq X \leqq I$ and $\operatorname{tr}(X)=k$ is convex and contains the projections of rank $k$.

There is another way of expressing Lemma 1.4 which seems of independent interest. Let $\mathscr{C}_{k}$ be the positive cone generated by the projections of rank $k$, i.e., $\mathscr{C}_{k}$ is the smallest set that contains all such projections and is closed with respect to addition and multiplication by non-negative scalars.

COROLlaRY. $\mathscr{C}_{k}=\{X \geqq 0: \operatorname{tr}(X) \geqq k\|X\|\}$.

Proof of Theorem 1.2. If $O \leqq X \leqq I$ and $\operatorname{tr}(X)=k$, then by Lemma $1.4 X=\sum_{i} a_{i} F_{i}$ is a convex combination of projections of rank $k$. Hence

$$
\frac{1}{k} \operatorname{tr}(X A)=\frac{1}{k} \sum_{i} a_{i} \operatorname{tr}\left(F_{i} A\right)
$$

is a convex combination of the points $(1 / k) \operatorname{tr}\left(F_{i} A\right) \in W_{k}(A)$. Using Berger's result that $W_{k}(A)$ is convex, we find that $(1 / k) \operatorname{tr}(X A) \in W_{k}(A)$. This proves one of the inclusions asserted in Theorem 1.2. The other is trivial.

THEOREM 1.5. If $A$ is a normal operator on $H$, then

$$
\operatorname{Ext} W_{k}(A) \subset\left\{\frac{1}{k}\left(\lambda_{i_{1}}+\lambda_{i_{2}}+\ldots+\lambda_{i_{k}}\right)\right\},
$$

the set of normalized $k$-fold sums of eigenvalues of $A$.

Proof. By the spectral theorem, we can suppose that $A=\sum_{i=1}^{n} \lambda_{i} E_{i}$, where the $E_{i}$ are mutually orthogonal projections of rank 1. Then

Suppose that $\lambda=(1 / k) \operatorname{tr}(P A)$ belongs to $W_{k}(A)$.

$$
\lambda=\frac{1}{k} \operatorname{tr}\left(P\left(\sum_{i} \lambda_{i} E_{i}\right)\right)=\frac{1}{k} \sum_{i} \lambda_{i} \operatorname{tr}\left(P E_{i}\right) .
$$

Since $\operatorname{tr}\left(P E_{i}\right)=\operatorname{tr}\left(P^{2} E_{i}\right)=\operatorname{tr}\left(P E_{i} P\right) \geqq 0$, the $n$-tuple with coordinates $\operatorname{tr}\left(P E_{i}\right)$ belongs to $P_{k}$. By Lemma 1.3 there are numbers $a_{i}, x_{i j}$ such that

$$
0 \leqq a_{i} \leqq 1, \quad \sum a_{i}=1, \quad \operatorname{tr}\left(P E_{j}\right)=\sum_{i} a_{i} x_{i j}
$$

where, for each $i$, exactly $k$ of the $x_{i j}$ are 1 and the others are 0 .

Hence

$$
\lambda=\frac{1}{k} \sum_{j} \lambda_{j} \operatorname{tr}\left(P E_{j}\right)=\frac{1}{k} \sum_{j} \lambda_{j}\left(\sum_{i} a_{i} x_{i j}\right)=\sum_{i} a_{i}\left(\frac{1}{k} \sum_{j} x_{i j} \lambda_{j}\right) .
$$

Now $(1 / k) \sum_{j} x_{i j} \lambda_{j}$ is, for each $i$, a normalized $k$-fold sum of eigenvalues of $A$. Thus each $\lambda \in W_{k}(A)$ is a convex combination of normalized $k$-fold sums of eigenvalues. Since these sums are in $W_{k}(A)$, the proof is complete. 
REMARK. Theorem 1.5 includes the known fact that the extreme points of the numerical range of a normal operator are eigenvalues. This is also true if $H$ is infinite-dimensional [6].

2. Diagonals of matrices. Our interest in $k$-numerical ranges arose from their connection with an unsolved problem in matrix theory. Given $n$ complex numbers $\lambda_{1}, \lambda_{2}, \ldots, \lambda_{n}$, the problem asks for necessary and sufficient conditions on $\mu_{1}, \mu_{2}, \ldots, \mu_{n}$ in order that there exist a normal matrix with eigenvalues $\lambda_{1}, \lambda_{2}, \ldots \lambda_{n}$ and main diagonal $\left\langle\mu_{1}, \mu_{2}, \ldots, \mu_{n}\right\rangle$. Equivalently, if $A$ is a given normal matrix, to determine which $n$-tuples $\left\langle\mu_{1}, \mu_{2}, \ldots \mu_{n}\right\rangle$ can serve as the diagonal of some matrix unitarily equivalent to $A$. Or again, to characterize the $n$-dimensional numerical range $\mathscr{W}(A)$ consisting of $n$-tuples of the form $\left\langle\left(A f_{1}, f_{1}\right),\left(A f_{2}, f_{2}\right), \ldots,\left(A f_{n}, f_{n}\right)\right\rangle$, where the $f_{i}$ form an orthonormal basis of $H$. In the case in which $A$ is Hermitian the problem was solved by Horn [3].

THEOREM 2.1. $\mathscr{W}(A)$ is arcwise connected for any matrix $A$.

Proof. If $e_{1}, e_{2}, \ldots, e_{n}$ is a fixed orthonormal basis of $H$, then any point in $\mathscr{W}(A)$ has the form

$$
\left\langle\left(U^{-1} A U e_{1}, e_{1}\right),\left(U^{-1} A U e_{2}, e_{2}\right), \ldots,\left(U^{-1} A U e_{n}, e_{n}\right)\right\rangle,
$$

where $U$ is a unitary operator on $H$. The theorem is therefore an immediate consequence of the well known fact that the group of unitary matrices is arcwise connected.

If $\lambda=\left\langle\lambda_{1}, \lambda_{2}, \ldots, \lambda_{n}\right\rangle$ is a complex $n$-tuple and $\pi$ is a permutation of the numbers $1,2, \ldots, n$, let $\lambda_{\pi}$ be the $n$-tuple $\left\langle\lambda_{\pi(1)}, \lambda_{\pi(2)}, \ldots, \lambda_{\pi(n)}\right\rangle$, and let $\mathscr{H}(\lambda)$ denote the convex hull of the vectors $\lambda_{\pi}$.

THEOREM 2.2. If $A$ is normal with eigenvalues $\lambda_{1}, \lambda_{2}, \ldots, \lambda_{n}$, then $\mathscr{H}(\lambda)=\mathscr{C} \mathscr{H}(A)$ (the convex hull of $\mathscr{W}(A))$.

Proof. Clearly each $\lambda_{\pi}$ belongs to $\mathscr{W}(A)$ and therefore $\mathscr{H}(\lambda) \subset \mathscr{C} \mathscr{W}(A)$. To complete the proof, it is enough to show that $\mathscr{W}(A) \subset \mathscr{H}(\lambda)$.

If $\mu \in \mathscr{W}(A)$, then there is an orthonormal basis $f_{1}, f_{2}, \ldots, f_{n}$ such that $\mu_{i}=\left(A f_{i}, f_{i}\right)$ for $i=1,2, \ldots, n$. If $e_{1}, e_{2}, \ldots, e_{n}$ are the eigenvectors of $A$ corresponding to $\lambda_{1}, \lambda_{2}, \ldots, \lambda_{n}$, then a computation shows that $\mu=P \lambda$, where $P_{i j}=\left|\left(f_{i}, e_{j}\right)\right|^{2}$. Since $P$ is clearly doubly stochastic, $\dagger$ it follows from a theorem of Birkhoff [3] that $\mu$ belongs to $\mathscr{H}(\lambda)$.

REMARK. Lerer [5] gives an example of a $3 \times 3$ unitary matrix $A$ with the property that $\mathscr{W}(A)$ is a proper subset of $\mathscr{H}(\lambda)$. Theorem 2.2 therefore implies that in general $\mathscr{W}(A)$ need not be convex.

THEOREM 2.3. Let $A$ be a normal matrix with eigenvalues $\lambda_{1}, \lambda_{2}, \ldots, \lambda_{n}$, and for $\mu \in \mathbf{C}^{n}$ let $C(\mu)$ denote the convex hull of the set of normal matrices with spectrum the set of coordinates of $\mu$. Then

$$
\left\{\operatorname{tr}\left(A B^{*}\right): B \in C(\mu)\right\}=(\mathscr{H}(\lambda), \mu) .
$$

† A matrix with non-negative entries is doubly stochastic if the sum of the entries in each row and column is 1 . 
Proof. Let $[\mu]$ be the diagonal matrix whose main diagonal is $\mu$. Then $(\operatorname{diag}(T), \mu)=$ $\operatorname{tr}\left(T[\mu]^{*}\right)$ for any matrix $T$. Hence by Theorem 2.2 ,

$$
\begin{aligned}
(\mathscr{H}(\lambda), \mu)=\mathscr{C}(\mathscr{W}(A), \mu) & =\mathscr{C}\left\{\left(\operatorname{diag}\left(U^{-1} A U\right), \mu\right): U \text { unitary }\right\} \\
& =\mathscr{C}\left\{\operatorname{tr}\left(U^{-1} A U[\mu]^{*}\right): U \text { unitary }\right\} \\
& =\mathscr{C}\left\{\operatorname{tr}\left(A\left(U[\mu] U^{-1}\right)^{*}\right): U \text { unitary }\right\} \\
& =\left\{\operatorname{tr}\left(A B^{*}\right): B \in C(\mu)\right\} .
\end{aligned}
$$

COROLlaRY 1 (F. John [4]). For any subset $\sigma$ of $\mathbf{R}^{n}$, let $C(\sigma)$ be the set of all Hermitian matrices $A$ with $\mathscr{H}(\lambda) \subset \sigma$. If $\sigma$ is compact and convex, so is $C(\sigma)$.

Proof. If $\sigma$ is the half-space $\left\{\xi \in \mathbf{R}^{n}:(\xi, \mu) \geqq r\right\}$, then by Theorem 2.3 a Hermitian matrix $A$ belongs to $C(\sigma)$ if and only if $\operatorname{tr}\left(A B^{*}\right) \geqq r$ for all matrices $B$ that are unitarily equivalent to $[\mu]$. From this description it is clear that $C(\sigma)$ is closed and convex. If $\sigma$ is compact and convex, it is an intersection of closed half-spaces, and $C(\sigma)$ is therefore an intersection of closed sets. Thus $C(\sigma)$ is closed and convex; because it is bounded it is compact.

Theorem 2.3 yields another proof of Theorem 1.5:

COROLlaRy 2. If $A$ is normal with eigenvalues $\lambda_{1}, \lambda_{2}, \ldots, \lambda_{n}$, then

$$
W_{k}(A)=\mathscr{C}\left\{\frac{1}{k}\left(\lambda_{i_{1}}+\lambda_{i_{2}}+\ldots+\lambda_{i_{k}}\right)\right\} \quad(k=1,2, \ldots, n) .
$$

Proof. $k W_{k}(A)=\mathscr{C}\{\operatorname{tr}(A P): P$ projection of rank $k\}$

$$
\begin{aligned}
& =\left\{\operatorname{tr}\left(A B^{*}\right): B \in C\left(\mu_{k}\right)\right\} \\
& =\left(\mathscr{H}(\lambda), \mu_{k}\right) \\
& =\mathscr{C}\left\{\left(\lambda_{\pi}, \mu_{k}\right): \pi \text { permutation }\right\},
\end{aligned}
$$

where $\mu_{k}$ is the vector with the first $k$ entries equal to 1 and the rest equal to 0 .

The next theorem indicates a connection between $k$-numerical ranges and diagonals, and includes several results of [1].

THEOREM 2.4. If $A$ is a matrix, then $\lambda \in W_{k}(A)$ if and only if $\mathscr{W}(A)$ contains a vector with at least $k$ coordinates equal to $\lambda$.

Proof. Without loss of generality we may suppose that $\lambda=0$. If $P$ is a projection, let $C_{P}(A)=\left.P A\right|_{P(H)}$ be the compression of $A$ to the range of $P$. A simple computation shows that $\operatorname{tr}(P A)=\operatorname{tr}\left(C_{p}(A)\right)$.

Now if $0 \in W_{k}(A)$, then there is a projection $P$ of rank $k$ such that $\operatorname{tr}(P A)=0$. The operator $C_{p}(A)$ then has trace 0 and hence, using a result from [1], we can choose an orthonormal basis $f_{1}, \ldots, f_{k}$ of $P(H)$ such that $\left(A f_{i}, f_{i}\right)=\left(C_{p}(A) f_{i}, f_{i}\right)=0$ for $i=1,2, \ldots, k$. If $f_{k+1}, \ldots, f_{n}$ form an orthonormal basis of $P(H)^{\perp}$, then the matrix of $A$ relative to the basis $f_{1}, f_{2}, \ldots, f_{n}$ has at least $k$ zeros on the main diagonal. 
3. Diagonals of Hermitian matrices. For the remainder of the paper $A$ will denote an $n \times n$ Hermitian matrix $(n \geqq 3)$ with eigenvalues $\lambda_{1} \leqq \lambda_{2} \leqq \ldots \leqq \lambda_{n}$ and corresponding unit eigenvectors $e_{1}, e_{2}, \ldots, e_{n}$.

LEMMA 3.1. If $\mu \in W(A)$, there is a unit vector $f$ such that $(A f, f)=\mu$ and

$$
k W_{k}\left(A_{1}\right)=k W_{k}(A) \cap\left((k+1) W_{k+1}(A)-\mu\right),
$$

where $A_{1}$ is the compression of $A$ to the orthogonal complement of $f$, and $k=1,2, \ldots, n-1$.

Proof. Choose the largest integer $i$ such that $\mu \in\left[\lambda_{i}, \lambda_{i+1}\right]$. Since $A$ is Hermitian there is a unit vector $f$ in the span of $e_{i}$ and $e_{i+1}$ with $(A f, f)=\mu$. Define

$$
\begin{aligned}
& \alpha_{k}=\max \left\{\sum_{j=1}^{k} \lambda_{j}, \sum_{j=1}^{k+1} \lambda_{j}-\mu\right\}, \\
& \beta_{k}=\min \left\{\sum_{j=n}^{n} \lambda_{j}, \sum_{j=n-k}^{n} \lambda_{j}-\mu\right\} .
\end{aligned}
$$

It will be shown that each side of the above equation is $\left[\alpha_{k}, \beta_{k}\right]$.

A real number $x$ belongs to the interval $\left[\alpha_{k}, \beta_{k}\right]$ if and only if

$$
\begin{gathered}
\sum_{1}^{k} \lambda_{J} \leqq x \leqq \sum_{n-k+1}^{n} \lambda_{j}, \\
\sum_{1}^{k+1} \lambda_{j} \leqq x+\mu \leqq \sum_{n-k}^{n} \lambda_{j} .
\end{gathered}
$$

Theorem 1.5 shows that these conditions are respectively equivalent to

This proves that

$$
x \in k W_{k}(A), x+\mu \in(k+1) W_{k+1}(A) \text {. }
$$

$$
\left[\alpha_{k}, \beta_{k}\right]=k W_{k}(A) \cap\left((k+1) W_{k+1}(A)-\mu\right) .
$$

Now $k W_{k}\left(A_{1}\right)$ is the set of all sums $\sum_{j=1}^{k}\left(A g_{j}, g_{j}\right)$ where $g_{1}, g_{2}, \ldots, g_{k}$ are orthonormal vectors in $\{f\}^{\perp}$. Hence clearly $k W_{k}\left(A_{1}\right) \subset k W_{k}(A)$. It is also clear that $\sum_{j=1}^{k}\left(A g_{j}, g_{j}\right)+(A f, f)$ belongs to $(k+1) W_{k+1}(A)$. Therefore

$$
k W_{k}\left(A_{1}\right) \subset k W_{k}(A) \cap\left((k+1) W_{k+1}(A)-\mu\right) .
$$

To prove the reverse inclusion, it suffices to prove that the numbers $\alpha_{k}$ and $\beta_{k}$ belong to

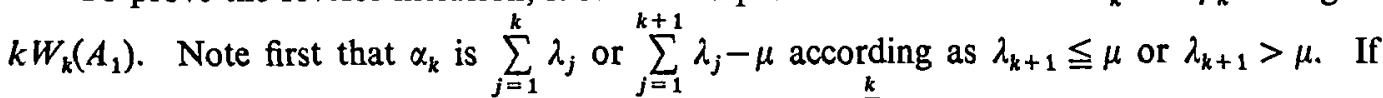
$\lambda_{k+1} \leqq \mu$ then $f$ is orthogonal to $e_{1}, \ldots, e_{k}$, and so $\alpha_{k}=\sum_{j=1}^{k} \lambda_{j} \in k W_{k}\left(A_{1}\right)$. If $\lambda_{k+1}>\mu$ then $f$ is in the span of $e_{1}, \ldots, e_{k+1}$; let $Q$ be the projection on this span and $P$ the projection on the orthogonal complement of $f$ in this span. Then $\operatorname{tr}(Q A)=\operatorname{tr}(P A)+\mu$ and $\operatorname{tr}(Q A)=\sum_{j=1}^{k+1} \lambda_{j}$, so 
that

$$
\operatorname{tr}\left(P A_{1}\right)=\operatorname{tr}(P A)=\sum_{j=1}^{k+1} \lambda_{j}-\mu=\alpha_{k}
$$

and $\alpha_{k} \in k W_{k}\left(A_{1}\right)$. The proof is completed by arguing similarly for $\beta_{k}$.

The last part of this proof can be based on the fact, proved in [7], that the spectrum of $A_{1}$ consists of $\lambda_{i}+\lambda_{i+1}-\mu$ and the points $\lambda_{j}$ with $j \neq i, i+1$.

It is now easy to obtain Horn's characterization of $\mathscr{W}(A)$.

THEOREM 3.2. Let $A$ be an $n \times n$ Hermitian matrix with eigenvalues $\lambda=\left\langle\lambda_{1}, \lambda_{2}, \ldots, \lambda_{n}\right\rangle$, and let $\mu=\left\langle\mu_{1}, \mu_{2}, \ldots, \mu_{n}\right\rangle$. The following are equivalent.

(i) $\mu \in \mathscr{H}(\lambda)$.

(ii) $\mu \in \mathscr{W}(A)$.

(iii) $\mu_{i_{1}}+\mu_{i_{2}}+\ldots+\mu_{i_{k}} \in k W_{k}(A)$

for each choice of subscripts and $k=1,2, \ldots, n$.

Proof. The equivalence of (i) and (ii) is an immediate consequence of Birkhoff's theorem (see [3]). Moreover, it is obvious that (ii) implies (iii). We show that (iii) implies (ii).

Choose a unit vector $f_{1}$ such that $\left(A f_{1}, f_{1}\right)=\mu_{1}$ as in Lemma 3.1, and let $A_{1}$ be the compression of $A$ to the orthogonal complement of $f_{1}$. If $j \geqq 2$, then $\mu_{1}+\mu_{j} \in 2 W_{2}(A)$; hence $\mu_{j} \in W\left(A_{1}\right)$ by Lemma 3.1. Also, if $j \neq k$ and $j, k \geqq 2$, then

$$
\mu_{j}+\mu_{k} \in\left(3 W_{3}(A)-\mu_{1}\right) \cap 2 W_{2}(A)=2 W_{2}\left(A_{1}\right) \text {. }
$$

The argument can now be repeated with $A_{1}$ replacing $A$. This gives a unit vector $f_{2}$ in $\left\{f_{1}\right\}^{\perp}$ such that $\left(A f_{2}, f_{2}\right)=\left(A_{1} f_{2}, f_{2}\right)=\mu_{2}$. Also, if $A_{2}$ is the compression of $A_{1}$ to $\left\{f_{1}\right\}^{\perp} \cap\left\{f_{2}\right\}^{\perp}$, then as before

$$
\begin{aligned}
\mu_{j} & \in W\left(A_{2}\right), \\
\mu_{j}+\mu_{k} & \in 2 W_{2}\left(A_{2}\right),
\end{aligned}
$$

for $j, k \geqq 3$ and $j \neq k$.

The proof is completed by $n-1$ repetitions of the same argument.

REMARK. We observed earlier that in general $\mathscr{W}(A) \neq \mathscr{H}(\lambda)$ for normal matrices. The reason for this is that Lemma 3.1 fails. For example, let $A$ be the $3 \times 3$ diagonal matrix with non-collinear eigenvalues $\lambda_{1}, \lambda_{2}, \lambda_{3}$, and let $\mu=\frac{2}{3} \lambda_{1}+\frac{1}{3} \lambda_{2}$. Let $f$ be any unit vector such that $(A f, f)=\mu$, and let $A_{1}$ be the compression of $A$ to $\{f\}^{\perp}$. If $z=\frac{1}{3} \lambda_{2}+\frac{2}{3} \lambda_{3}$, then $z$ belongs to $W_{1}(A) \cap\left(2 W_{2}(A)-\mu\right)$. However, it is easy to see that $W\left(A_{1}\right)$ is the line segment $\left[\frac{1}{3} \lambda_{1}+\frac{2}{3} \lambda_{2}, \lambda_{3}\right]$ (see [7] for example) and this does not contain $z$.

\section{REFERENCES}

1. P. A. Fillmore, On similarity and the diagonal of a matrix, Amer. Math. Monthly 76 (1969), 167-169.

2. P. R. Halmos, A Hilbert Space Problem Book (Princeton, 1967). 
3. Alfred Horn, Doubly stochastic matrices and the diagonal of a rotation matrix, Amer. J. Math. 76 (1954), 620-630.

4. F. John, On symmetric matrices whose eigenvalues satisfy linear inequalities, Proc. Amer. Math. Soc. 17 (1966), 1140-1146.

5. L. E. Lerer, On the diagonal elements of normal matrices (Russian), Mat. Issled. 2 (1967), 156-163.

6. C. R. MacCluer, On extreme points of the numerical range of normal operators, Proc. Amer. Math. Soc. 16 (1965), 1183-1184.

7. J. P. Williams, On compressions of matrices, J. London Math. Soc. 3 (1971), 526-530.

INDIANA UNIVERSITY

BLOOMINGTON

INDIANA

U.S.A. 